CONFORMAL GEOMETRY AND DYNAMICS

An Electronic Journal of the American Mathematical Society

Volume 12, Pages 97-108 (July 21, 2008)

S 1088-4173(08)00180-X

\title{
REAL SADDLE-NODE BIFURCATION FROM A COMPLEX VIEWPOINT
}

\author{
MICHAŁ MISIUREWICZ AND RODRIGO A. PÉREZ
}

\begin{abstract}
During a saddle-node bifurcation for real analytic interval maps, a pair of fixed points, attracting and repelling, collide and disappear. From the complex point of view, they do not disappear, but just become complex conjugate. The question is whether those new complex fixed points are attracting or repelling. We prove that this depends on the Schwarzian derivative $S$ at the bifurcating fixed point. If $S$ is positive, both fixed points are attracting; if it is negative, they are repelling.
\end{abstract}

\section{INTRODUCTION}

For the family of double standard maps,

$$
f_{a, b}(x)=2 x+a+\frac{b}{\pi} \sin (2 \pi x) \quad(\bmod 1)
$$

( $a$ is taken modulo 1 and $b$ varies from 0 to 1 ) introduced in [3], the values of the parameters with an attracting periodic orbit are grouped into cusp-like sets, called tongues. Let $T$ be the tongue of parameters for which there is an attracting fixed point. When the parameter $a$ varies, the graph of $f_{a, b}$ moves up or down, so starting inside $T$, we leave via a classical saddle-node bifurcation. This means that a pair of fixed points, an attracting one and a repelling one, collide and disappear (see Figure 2). But do they indeed disappear? From the complex viewpoint they simply go off the real line.

Complexification of the family of double standard maps was studied by Fagella and Garijo [1. It turns out that when we take into account complex attracting fixed points, $T$ develops a big bulb at the end of the tongue. Similar shapes, in other families, have been reported by many authors (see for instance, [2] or [4]).

Figure 1 illustrates the bulb at the tip of $T$. The real tongue is not shaded in black in order to distinguish it from the bulb, yet it is clear from the picture that they touch each other. In particular, stepping off the tip of $T$ makes both complex fixed points attracting; see Example 1.4 for a computationally simple family of this type.

On the other hand, for quadratic maps such behavior is impossible (see Example 1.3), since for a real quadratic polynomial any attracting fixed point must be real in order to attract the orbit of the unique critical point.

Received by the editors December 12, 2007.

2000 Mathematics Subject Classification. Primary 37E05, 37H20, 37F99.

Key words and phrases. Saddle-node, Schwarzian derivative, parabolic point.

The first author was partially supported by NSF grant DMS 0456526 .

The second author was partially supported by NSF grant DMS 0701557. 


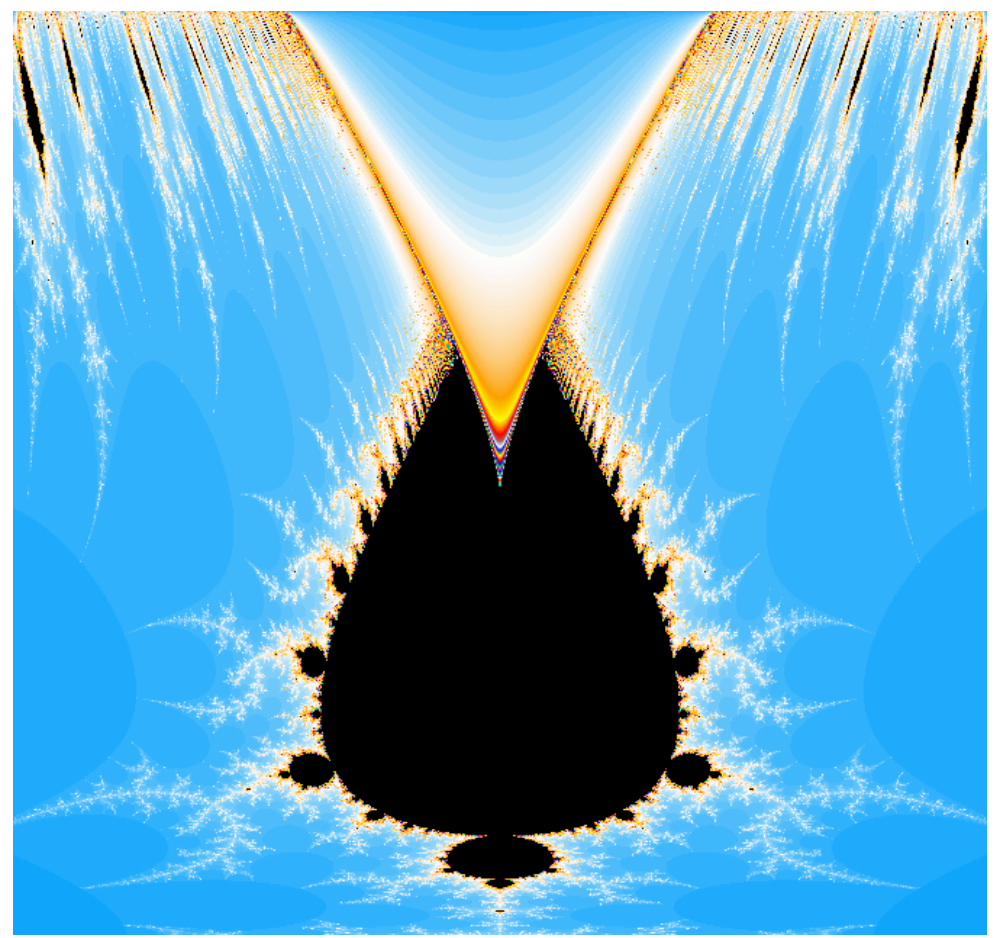

Figure 1. The parameter plane of complex double standard maps, with horizontal $a$-axis, and vertical $b$-axis.

The aim of this paper is to investigate the distinction between these two cases.

Throughout the paper, $f$ will be a real analytic map defined in a neighborhood of 0 , with $f(0)=0, f^{\prime}(0)=1$, and $f^{\prime \prime}(0)>0$, so 0 is a parabolic fixed point of multiplicity 2. For $r>0$, let $D_{r}$ be the open disk of radius $r$, centered at 0 , and let $C_{r}$ be its boundary. Abusing notation, we denote the complexification of $f$ also by $f$. Then, if its power series expansion at 0 is

$$
f(z)=\sum_{n=1}^{\infty} a_{n} z^{n},
$$

we have $a_{1}=1, a_{2}>0$, and $a_{n} \in \mathbb{R}$ for all $n$. Fix a positive number $R$, smaller than the radius of convergence of $f$. Then $f$ belongs to the space $\mathcal{A}_{R}$ of all analytic functions defined in a neighborhood of $\overline{D_{R}}$, taking real values on $D_{R} \cap \mathbb{R}$.

Because $f: \mathbb{R} \longrightarrow \mathbb{R}$ is convex, a small perturbation $f-\lambda^{2}$ with real $\lambda \neq 0$ has two real fixed points, one attracting and one repelling. For $f+\lambda^{2}$, the fixed point at 0 vanishes instead of bifurcating; see Figure 2. This is a standard saddle-node bifurcation, except that we use parameter $\lambda^{2}$ rather than $\lambda$.

In $\mathbb{C}$, on the other hand, $f_{\lambda}:=f+\lambda^{2}$ still has a pair of fixed points near 0 . Since the coefficients $a_{n}$ are real, the fixed points are complex conjugate and have the same type (attracting - neutral - repelling). The following proposition gives an analytical condition to determine this type in the family $f_{\lambda}$, and will serve as a model for our main result, Theorem 1.2 . 

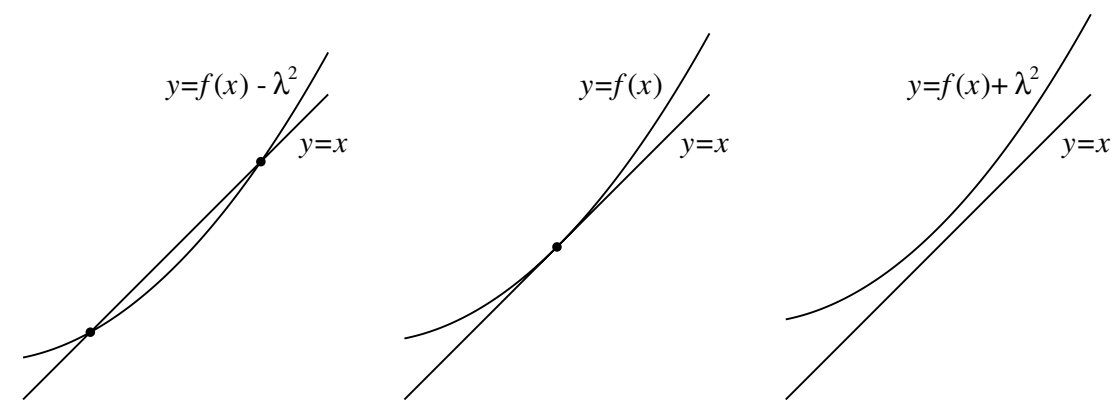

Figure 2. A saddle-node bifurcation.

Proposition 1.1. Let $z_{ \pm}$be the conjugate fixed points of $f_{\lambda}$ (for $\lambda$ real and small) near 0 . The absolute values of the multipliers at $z_{ \pm}$are

$$
\left|\left(f_{\lambda}\right)^{\prime}\left(z_{ \pm}\right)\right|=1+2\left(a_{2}-\frac{a_{3}}{a_{2}}\right) \lambda^{2}+O\left(\lambda^{3}\right)
$$

If $\lambda$ is sufficiently small, $z_{ \pm}$are both attracting or both repelling according to the sign of $a_{2}-\left(a_{3} / a_{2}\right)$. Note that the Schwarzian derivative of $f$ at 0 is $S f(0)=$ $6\left(a_{3}-a_{2}^{2}\right)=-6 a_{2}\left(a_{2}-a_{3} / a_{2}\right)$. Thus, the sign of $S f(0)$ rules the fixed point type for small constant perturbations. Connections between the sign of the Schwarzian at a bifurcating fixed point and the type of bifurcation have been described for some other types of bifurcations; see for example [5], where the period doubling bifurcation is considered. To generalize Proposition 1.1 to as large a space of perturbations as possible, we need some definitions.

For all $r>0$ and $h \in \mathcal{A}_{r}$ we denote by $\|h\|_{r}$ the supremum of $|h|$ on the closure of the disk $D_{r}$. Clearly, $\|h\|_{r}<\infty$. For a given $\varepsilon>0$, let $\mathcal{P}_{\varepsilon, R}(f)$ be the space of all functions $\tilde{f} \in \mathcal{A}_{R}$ such that $\|f-\tilde{f}\|_{R}<\varepsilon$.

If $\varepsilon$ is small enough, and $\tilde{f} \in \mathcal{P}_{\varepsilon, R}(f)$, there is a small real number $c \in D_{R}$ such that $\widetilde{f}^{\prime}(c)=1$. Precise conditions on $\varepsilon$ will be given in Section 4 , Then, if $\widetilde{f}(c)>c$, the map $\tilde{f}$ has no real fixed points near 0 . We say in this case that $\tilde{f}$ has complex nature.

Our main result characterizes the type of the bifurcated fixed points of an arbitrary perturbation $\tilde{f}$ of $f$ with complex nature.

Theorem 1.2. There exist $r \in(0, R)$ and $\varepsilon>0$ such that every function $\tilde{f} \in$ $\mathcal{P}_{\varepsilon, R}(f)$ with complex nature has exactly two fixed points in $D_{r}$. If $S f(0)>0$, they are both attracting, and if $S f(0)<0$, they are both repelling.

Example 1.3. Set $f(z)=z^{2}+z$, and consider the family $f_{\varepsilon}(z)=f(z)+\varepsilon(\varepsilon>0)$. The fixed points of $f_{\varepsilon}$ are $\pm \sqrt{\varepsilon} i$, and since $f_{\varepsilon}^{\prime}(z)=2 z+1$, both fixed points are repelling. In this case, $S f(0)=6\left(a_{3}-a_{2}^{2}\right)=-6$.

Example 1.4. Now let $f(z)=2 z^{3}+z^{2}+z$, and $f_{\varepsilon}(z)=2 z^{3}+z^{2}+z+(1+2 z) \varepsilon$. As $\varepsilon$ increases, the fixed point at 0 bifurcates again into the pair $\pm \sqrt{\varepsilon} i$. This time however, $f_{\varepsilon}^{\prime}(z)=6 z^{2}+2 z+(1+2 \varepsilon)$, so the multipliers are $1-4 \varepsilon \pm 2 \sqrt{\varepsilon} i$, and the fixed points are attracting for small enough $\varepsilon>0$. In this case, $S f(0)=6\left(a_{3}-a_{2}^{2}\right)=6$. 


\section{Preliminaries}

For ease of reference, we collect here the basic facts and definitions that are used in the proof. In this section, $\varphi$ and $\psi$ are functions from $\mathcal{A}_{r}$ for some $r>0$, and

$$
\varphi(z)=\sum_{n=0}^{\infty} c_{n} z^{n} .
$$

2.1. Cauchy estimates. If $\|\varphi\|_{r} \leq \varepsilon$, the derivatives satisfy $\left|\varphi^{(n)}(0)\right| \leq \frac{n ! \varepsilon}{r^{n}}$. It follows that the power series coefficients of $\varphi$ satisfy

$$
\left|c_{n}\right| \leq \frac{\varepsilon}{r^{n}} .
$$

If $0<r^{\prime}<r$, the same estimate, applied in all disks of radius $r-r^{\prime}$ centered at points of $D_{r^{\prime}}$, shows that

$$
\left\|\varphi^{(n)}\right\|_{r^{\prime}} \leq \frac{n ! \varepsilon}{\left(r-r^{\prime}\right)^{n}}
$$

2.2. Tail estimates. Define the $n^{\text {th }}$ order tail of $\varphi$ as the function $\varphi^{[n]}$ that satisfies

$$
\varphi(z)=\left(\sum_{k=0}^{n-1} c_{k} z^{k}\right)+z^{n} \cdot \varphi^{[n]}(z) .
$$

Lemma 2.1. Assume that $\|\varphi\|_{r}<\varepsilon$. Then for all $n$ we have

$$
\left\|\varphi^{[n]}\right\|_{r}<\left(\frac{2}{r}\right)^{n} \varepsilon
$$

Proof. The disk $D_{r}$ contains 0, so $|\varphi(0)|<\varepsilon$. Together with $|\varphi(z)|=\mid \varphi(0)+z$. $\varphi^{[1]}(z) \mid<\varepsilon$, this implies $\left|z \cdot \varphi^{[1]}(z)\right|<2 \varepsilon$, so $\left|\varphi^{[1]}(z)\right|<\frac{2 \varepsilon}{|z|}$ for all $z \neq 0$. However, $\varphi^{[1]}$ attains its maximum on the boundary of the disk, so $\left\|\varphi^{[1]}\right\|_{r}<\frac{2}{r} \varepsilon$. Since $\left(\varphi^{[n]}\right)^{[1]}=\varphi^{[n+1]}$, it follows by induction that $\left\|\varphi^{[n]}\right\|_{r}<\left(\frac{2}{r}\right)^{n} \varepsilon$.

2.3. Bound on the difference of square roots. If $\varphi(0) \neq 0$, then $\varphi$ has two well-defined square root branches around 0 and their radius of convergence is $\min \{|z|: \varphi(z)=0\}>0$.

Lemma 2.2. Let $\varphi, \psi \in \mathcal{A}_{r}$. Assume that there is $\Delta>0$ such that $\|\varphi-\psi\|_{r}<\Delta$ and $|\varphi(z)|>\Delta,|\psi(z)|>0$ for all $z \in D_{r}$. Let $\sqrt{\psi}$ be a branch of the square root of $\psi$ in $D_{r}$. Then there is a branch $\sqrt{\varphi}$ of the square root of $\varphi$ in $D_{r}$, such that $\|\sqrt{\varphi}-\sqrt{\psi}\|_{r}<\sqrt{\Delta}$.

Proof. Fix a branch $\eta$ of the square root of $\varphi$ in $D_{r}$. For every $z \in D_{r}$ we have

$$
|\eta(z)-\sqrt{\psi}(z)| \cdot|\eta(z)+\sqrt{\psi}(z)|=|\varphi(z)-\psi(z)|<\Delta,
$$

so either $|\eta(z)-\sqrt{\psi}(z)|<\sqrt{\Delta}$ or $|\eta(z)+\sqrt{\psi}(z)|<\sqrt{\Delta}$.

The sets of the points where each of those inequalities holds, are open. If both sets were nonempty, there would be a point $z \in D_{r}$ at which both inequalities hold. Then

$$
2|\eta(z)| \leq|\eta(z)-\sqrt{\psi}(z)|+|\eta(z)+\sqrt{\psi}(z)|<2 \sqrt{\Delta},
$$

which contradicts $|\varphi(z)|>\Delta$. Thus, one of the inequalities holds for every $z \in D_{r}$, and we choose as $\sqrt{\varphi}$ either $\eta$, if the first inequality holds, or $-\eta$, if the second inequality holds. 


\subsection{Bound on the difference of inverses.}

Lemma 2.3. Suppose that $\varphi^{\prime}$ is bounded away from 0 in the sense that there is a complex number $a \neq 0$ such that $\left|\varphi^{\prime}(z)-a\right|<|a| / 2$ for every $z \in D_{r}$. Then for every distinct $w, z \in D_{r}$ we have

$$
|\varphi(z)-\varphi(w)|>\frac{|a|}{2} \cdot|z-w| .
$$

Proof. Consider the segment joining $w$ with $z$, parametrized by $\gamma(t)=w+t(z-w)$ $(0 \leq t \leq 1)$. By the chain rule,

$$
\varphi(z)-\varphi(w)=\varphi(\gamma(1))-\varphi(\gamma(0))=\int_{0}^{1}(\varphi \circ \gamma)^{\prime}(t) d t=\int_{0}^{1} \varphi^{\prime}(\gamma(t)) \cdot(z-w) d t
$$

so

$$
|\varphi(z)-\varphi(w)-a \cdot(z-w)|=\mid \int_{0}^{1}\left(\left(\varphi^{\prime}(\gamma(t))-a\right) d t|\cdot| z-w\left|<\frac{|a|}{2} \cdot\right| z-w \mid .\right.
$$

Thus,

$$
|\varphi(z)-\varphi(w)|>|a \cdot(z-w)|-\frac{|a|}{2} \cdot|z-w|=\frac{|a|}{2} \cdot|z-w| .
$$

Now assume that $\varphi$ and $\psi$ satisfy the conditions of Lemma 2.3 with the same $a$. In particular, this implies that $\varphi$ and $\psi$ are univalent. The following result gives a bound on the difference of the inverses $\varphi^{-1}, \psi^{-1}$ when $\varphi$ and $\psi$ are close.

Lemma 2.4. If $\varphi$ and $\psi$ satisfy the conditions of Lemma 2.3 with the same $a$ and $\|\varphi-\psi\|_{r}<|a| r / 4$, then $\varphi\left(D_{r / 2}\right) \subset \psi\left(D_{r}\right)$. Moreover, if the radius $s>0$ is such that $D_{s} \subset \varphi\left(D_{r / 2}\right)$, then $\left\|\varphi^{-1}-\psi^{-1}\right\|_{s} \leq r / 2$.

Proof. Fix a point $z \in D_{r / 2}$ and denote by $C \subset D_{r}$ the circle of radius $r / 2$ centered at $z$. Then the winding number of $\varphi(C)$ around $\varphi(z)$ is 1 . By Lemma 2.3 and the bound on $\varphi-\psi$, all points $w \in C$ satisfy

$$
|\varphi(z)-\varphi(w)|>\frac{|a|}{2} \cdot|z-w|=\frac{|a| r}{4}>|\varphi(w)-\psi(w)|,
$$

so the winding number of $\psi(C)$ around $\varphi(z)$ is also 1 . Hence, there exists a point $\zeta$ in the disk bounded by $C$, such that $\psi(\zeta)=\varphi(z)$. This proves that $\varphi\left(D_{r / 2}\right) \subset \psi\left(D_{r}\right)$. Moreover, $|\zeta-z|<r / 2$, so taking the supremum over all $z$ such that $\varphi(z) \in D_{s}$, yields

$$
\left\|\varphi^{-1}-\psi^{-1}\right\|_{s} \leq r / 2
$$

\section{Proof of the Proposition}

The idea of the proof is to compute an expansion of the fixed points of $f_{\lambda}$ as power series of $\lambda$. The resulting formula (3.3) can then be inserted in the expansion of $f_{\lambda}^{\prime}$ to express the multipliers, and then their absolute values, as power series in $\lambda$.

Proof of Proposition 1.1. The fixed points of $f_{\lambda}$ satisfy the equation

$$
\lambda^{2}=-z^{2} \cdot f^{[2]}(z)=-\sum_{n=2}^{\infty} a_{n} z^{n} .
$$

Since $a_{2}>0$, the square root of $f^{[2]}$ has a well-defined branch in a neighborhood of 0 ; see Subsection 2.3;

$$
\sqrt{f^{[2]}}=\sqrt{a_{2}}+O(z) .
$$


Thus, the fixed points of $f_{\lambda}$ are roots of the equation

$$
\lambda=\left( \pm i \sqrt{a_{2}}\right) z+O\left(z^{2}\right),
$$

and since the right-hand side is an analytic function with value 0 at 0 and non-zero derivative at 0 , it can be solved for $z_{ \pm}$in terms of $\lambda$ in a neighborhood of 0 :

$$
z_{ \pm}=A \lambda+B \lambda^{2}+O\left(\lambda^{3}\right) .
$$

The coefficients $A$ and $B$ can be determined by substituting (3.2) into (3.1):

$$
\begin{aligned}
\lambda^{2} & =-\sum_{n=2}^{\infty} a_{n}\left(A \lambda+B \lambda^{2}+O\left(\lambda^{3}\right)\right)^{n} \\
& =\left(-a_{2} A^{2}\right) \lambda^{2}+\left(-2 a_{2} A B-a_{3} A^{3}\right) \lambda^{3}+O\left(\lambda^{4}\right) .
\end{aligned}
$$

Comparing the corresponding coefficients, we find that

$$
A= \pm \frac{i}{\sqrt{a_{2}}}, \quad B=\frac{a_{3}}{2 a_{2}^{2}},
$$

and thus, the two fixed points of $f_{\lambda}$ are

$$
z_{ \pm}=z_{ \pm}(\lambda)= \pm \frac{i}{\sqrt{a_{2}}} \lambda+\frac{a_{3}}{2 a_{2}^{2}} \lambda^{2}+O\left(\lambda^{3}\right) .
$$

We substitute (3.3) into the formula for $f_{\lambda}^{\prime}$ to find the fixed point multipliers:

$$
\begin{aligned}
f_{\lambda}^{\prime}\left(z_{ \pm}(\lambda)\right)= & 1+2 a_{2}\left( \pm \frac{i}{\sqrt{a_{2}}} \lambda+\frac{a_{3}}{2 a_{2}^{2}} \lambda^{2}+O\left(\lambda^{3}\right)\right) \\
& +3 a_{3}\left( \pm \frac{i}{\sqrt{a_{2}}} \lambda+\frac{a_{3}}{2 a_{2}^{2}} \lambda^{2}+O\left(\lambda^{3}\right)\right)^{2}+O\left(\lambda^{3}\right) \\
= & 1 \pm\left(2 i \sqrt{a_{2}}\right) \lambda-\left(\frac{2 a_{3}}{a_{2}}\right) \lambda^{2}+O\left(\lambda^{3}\right) .
\end{aligned}
$$

By the triangle inequality

$$
|| f_{\lambda}^{\prime}\left(z_{ \pm}\right)|-| 1 \pm\left(2 i \sqrt{a_{2}}\right) \lambda-\left(\frac{2 a_{3}}{a_{2}}\right) \lambda^{2}||<O\left(\lambda^{3}\right),
$$

so the absolute values of the multipliers at the fixed points are approximated to third order by $\left|1 \pm\left(2 i \sqrt{a_{2}}\right) \lambda-\left(\frac{2 a_{3}}{a_{2}}\right) \lambda^{2}\right|$. Since

$$
\begin{aligned}
& \left|1 \pm\left(2 i \sqrt{a_{2}}\right) \lambda-\left(\frac{2 a_{3}}{a_{2}}\right) \lambda^{2}\right|^{2}=\left|\left(1-\left(\frac{2 a_{3}}{a_{2}}\right) \lambda^{2}\right) \pm i\left(2 \sqrt{a_{2}} \lambda\right)\right|^{2} \\
& \quad=\left(1-\frac{2 a_{3}}{a_{2}} \lambda^{2}\right)^{2}+\left(2 \sqrt{a_{2}} \lambda\right)^{2}=1+4\left(a_{2}-\frac{a_{3}}{a_{2}}\right) \lambda^{2}+O\left(\lambda^{4}\right),
\end{aligned}
$$

the absolute value of the multipliers is approximated by the square root of (3.4) up to order three:

$$
\left|f_{\lambda}^{\prime}\left(z_{ \pm}\right)\right|=1+2\left(a_{2}-\frac{a_{3}}{a_{2}}\right) \lambda^{2}+O\left(\lambda^{3}\right) .
$$

\section{Smallness COnditions}

Theorem 1.2 claims the existence of "small enough" $\varepsilon$ and $r$ for which the conclusion holds. In this section we describe a sufficient set of smallness conditions on $\varepsilon$ and $r$ that make the result true. Each condition is expressed in terms of the original map $f$. Moreover, conditions on $\varepsilon$ may depend on the value of $r$, but not the other way. This prevents a logical loop.

The first condition is needed to characterize those perturbations of $f$ that have a bifurcated pair of complex fixed points. 
Lemma 4.1. There exists $r_{0} \in(0, R)$ such that for every $r \in\left(0, r_{0}\right]$ there exists $\varepsilon>0$ such that if $\tilde{f} \in \mathcal{P}_{\varepsilon, R}(f)$, then

(a) $\widetilde{f}(z)-z$ has exactly two zeros (counting multiplicities) in $D_{r}$,

(b) $\tilde{f}^{\prime}-1$ has exactly one zero $c \in D_{r}$, and this zero is real,

(c) $\tilde{f}^{\prime \prime}(c)>0$.

Proof. Set $h(z)=f(z)-z$. Then $h(0)=h^{\prime}(0)=0$ and $h^{\prime \prime}(0)>0$. Therefore, there exists $r_{0} \in(0, R)$ such that for every $r \in\left(0, r_{0}\right]$ the winding number of $h\left(C_{r}\right)$ around 0 is 2 and the winding number of $h^{\prime}\left(C_{r}\right)$ around 0 is 1 . If $\widetilde{f} \in \mathcal{P}_{\varepsilon, R}(f)$, then for $\widetilde{h}(z)=\widetilde{f}(z)-z$ we have

$$
\|\widetilde{h}-h\|_{R}<\varepsilon .
$$

Thus, if $\varepsilon$ is sufficiently small, then the winding number of $\widetilde{h}\left(C_{r}\right)$ around 0 is 2, and thus $\widetilde{h}$ has exactly two zeros in $D_{r}$. This proves (a). By (2.2) and (4.1), $\left\|\widetilde{h}^{\prime}-h^{\prime}\right\|_{r}<\varepsilon /(R-r)$, so if $\varepsilon$ is sufficiently small, the winding number of $\widetilde{h}^{\prime}\left(C_{r}\right)$ around 0 is 1 . Since $\bar{c}$ is also a zero of $\widetilde{h}^{\prime}, c$ must be real. This proves (b). Finally, by (2.2) and (4.1), $\left\|\widetilde{h}^{\prime \prime}-h^{\prime \prime}\right\|_{r}<2 \varepsilon /(R-r)^{2}$, so if $\varepsilon$ is sufficiently small, then $\operatorname{Re}\left(\tilde{f}^{\prime \prime}(z)\right)=\operatorname{Re}\left(\widetilde{h}^{\prime \prime}(z)\right)>0$ for all $z \in D_{r}$. Since $c$ is real and belongs to $D_{r}$, we get (c).

Now, the first condition for $r$ and $\varepsilon$ is:

$$
r_{0}, r \text { and } \varepsilon \text { are as in Lemma 4.1, }
$$

Note that for a given $r$ we can make $\varepsilon$ smaller without violating this condition.

Let $\widetilde{f}$ and $c$ be as in Lemma 4.1. If $\widetilde{f}(c)-c>0$, we say that $\widetilde{f}$ has complex nature, and set

Set

$$
\Lambda:=\sqrt{\widetilde{f}(c)-c}
$$

$$
\xi(\varepsilon):=2 \sup _{\tilde{f} \in \mathcal{P}_{\varepsilon, R}(f)}|c| .
$$

By Lemma 4.1(b), for every $r>0$ there is $\varepsilon>0$ such that if $\tilde{f} \in \mathcal{P}_{\varepsilon, R}(f)$, then $|c|<r$. Therefore $\xi$ is a finite monotone function of $\varepsilon$ with

$$
\lim _{\varepsilon \rightarrow 0} \xi(\varepsilon)=0 .
$$

We require

$$
\xi(\varepsilon)<r<\frac{R}{2}
$$

Set

$$
\delta:=2\left(\xi(\varepsilon) \cdot\left(\left\|f^{\prime}\right\|_{R}+2 \varepsilon / R+1\right)+\varepsilon\right) .
$$

Note that by (4.3), $\delta=\delta(\varepsilon)$ goes to 0 when $\varepsilon \rightarrow 0$.

Now we list several conditions that tell us how small $r$ should be. Since $a_{2} \neq 0$, the radius $r$ can be chosen small enough that

$$
\inf _{z \in D_{r}}\left|f^{[2]}(z)\right|>0 \text {. }
$$


Then there are two well-defined branches of the square root of $f^{[2]}$. Notice that $\left(z \cdot \sqrt{f^{[2]}(z)}\right)_{\mid z=0}^{\prime}= \pm \sqrt{a_{2}}$, where the sign depends on the choice of a branch. Let $\sqrt{f^{[2]}}$ be the branch that makes the derivative equal to $\sqrt{a_{2}}$. Let $r$ be small enough that

$$
\sup _{z \in D_{r}}\left|\left(z \cdot \sqrt{f^{[2]}(z)}\right)^{\prime}-\sqrt{a_{2}}\right|<\frac{\sqrt{a_{2}}}{2} .
$$

Similarly, since $\sqrt{f^{[2]}(0)}=\sqrt{a_{2}}$, we may assume that $r$ is small enough, so that

$$
\left\|\sqrt{f^{[2]}}-\sqrt{a_{2}}\right\|_{r}<\frac{\sqrt{a_{2}}}{8} .
$$

We also may assume that $r$ is small enough, so that

$$
r \cdot\left\|\left(\sqrt{f^{[2]}}\right)^{\prime}\right\|_{r}<\frac{\sqrt{a_{2}}}{8} .
$$

Now that $r$ is fixed, we list several conditions that tell us how small $\varepsilon$ should be. Remember that if $\varepsilon$ is small, $\delta$ is also small.

Using Lemmas 2.1 and 2.2, we see that we can require $\varepsilon$ to be small enough, so that

$$
\left\|\sqrt{g^{[2]}}-\sqrt{a_{2}}\right\|_{r}<\left\|\sqrt{f^{[2]}}-\sqrt{a_{2}}\right\|_{r}+\frac{\sqrt{a_{2}}}{8}
$$

and

$$
r \cdot\left\|\left(\sqrt{g^{[2]}}\right)^{\prime}\right\|_{r}<r \cdot\left\|\left(\sqrt{f^{[2]}}\right)^{\prime}\right\|_{r}+\frac{\sqrt{a_{2}}}{8}
$$

for every $g \in \mathcal{P}_{\varepsilon, R}(f)$ and one of the branches of $\sqrt{g^{[2]}}$.

Condition (C3) can be strengthened by letting $\varepsilon$ be small enough, so that

$$
\inf _{z \in D_{r}} \frac{\left|f^{[2]}(z)\right|}{2}>\frac{4 \delta}{r^{2}} .
$$

The coefficient $a_{2}$ is fixed beforehand. We require $\varepsilon$ to be small enough, so that

$$
2 \sqrt{\delta}<\frac{\sqrt{a_{2}} r}{4}
$$

Finally, we require $\varepsilon$ to be small enough, so that if $g(z)=\sum_{n=1}^{\infty} c_{n} z^{n}$ stands for an arbitrary function in $\mathcal{P}_{\varepsilon, R}(f)$, then

$$
c_{2}>\frac{a_{2}}{2}
$$




\section{Proof of the Theorem}

Let $\varepsilon, r$ satisfy conditions ([C1) $-(\mathbf{C} 11)$, and select a function $\widetilde{f} \in \mathcal{P}_{\varepsilon, R}(f)$ with complex nature (i.e., $\left.\Lambda^{2}=\widetilde{f}(c)-c>0\right)$.

Consider the functions $g_{\lambda}(z):=\lambda^{2}+\widetilde{f}(z+c)-\widetilde{f}(c)$ with $\lambda \in[0, \Lambda]$. We will show that the elements of this family are uniformly close to $f$.

Lemma 5.1. Regardless of the choice of $\widetilde{f} \in \mathcal{P}_{\varepsilon, R}(f)$, the family $\left\{g_{\lambda}\right\}_{\lambda \in[0, \Lambda]}$ is contained in $\mathcal{P}_{\delta, r}(f)$, where $\delta$ is as in (4.4).

Proof. First we estimate $\left\|g_{\Lambda}-\widetilde{f}\right\|_{r}$. By Lemma 2.1 we have

$$
\left\|\tilde{f}^{\prime}-f^{\prime}\right\|_{r} \leq\left\|\tilde{f}^{\prime}-f^{\prime}\right\|_{R}<\frac{2 \varepsilon}{R} .
$$

Moreover, $g_{\Lambda}(z)=\tilde{f}(z+c)-c$. Therefore,

$$
\begin{aligned}
\left\|g_{\Lambda}-\tilde{f}\right\|_{r} & \leq \sup _{z \in D_{r}}|\tilde{f}(z+c)-\tilde{f}(z)|+|c| \\
& \leq|c| \cdot\left\|\tilde{f}^{\prime}\right\|_{r}+|c|<\xi(\varepsilon) \cdot\left(\left\|f^{\prime}\right\|_{R}+2 \varepsilon / R+1\right) .
\end{aligned}
$$

Then we obtain a uniform bound for $g_{\Lambda}$ :

$$
\left\|g_{\Lambda}-f\right\|_{r} \leq\left\|g_{\Lambda}-\tilde{f}\right\|_{r}+\|\tilde{f}-f\|_{R}<\xi(\varepsilon) \cdot\left(\left\|f^{\prime}\right\|_{R}+2 \varepsilon / R+1\right)+\varepsilon .
$$

In particular, the right-hand expression above is a bound for $\Lambda^{2}=g_{\Lambda}(0)$, since $f(0)=0$. The uniform bound for all $g_{\lambda}$ now follows from the above inequality since $\left\|g_{\lambda}-g_{\Lambda}\right\|_{r}=\Lambda^{2}-\lambda^{2} \leq \Lambda^{2}$ :

$$
\begin{aligned}
\left\|g_{\lambda}-f\right\|_{r} & \leq\left\|g_{\lambda}-g_{\Lambda}\right\|_{r}+\left\|g_{\Lambda}-f\right\|_{r} \\
& \leq 2\left(\xi(\varepsilon) \cdot\left(\left\|f^{\prime}\right\|_{R}+2 \varepsilon / R+1\right)+\varepsilon\right)=\delta .
\end{aligned}
$$

Note that $\delta$ depends on $\varepsilon$, but not on the choice of $\widetilde{f}$. Moreover, $\Lambda$ is uniformly bounded.

Observe that $g_{0}(0)=0, g_{0}^{\prime}(0)=1, g_{0}^{\prime \prime}(0)>0$, and $g_{\lambda}=g_{0}+\lambda^{2}$. In particular, $g_{\lambda}$ has complex nature for all $\lambda \in(0, \Lambda]$. Thus, Proposition 1.1 applies to the family $\left\{g_{\lambda}\right\}_{\lambda \in[0, \Lambda]}$. Note also that $g_{\Lambda}(z)=\widetilde{f}(z+c)-c$; i.e., $g_{\Lambda}$ is smoothly conjugate to $\widetilde{f}$. This implies that the fixed point multipliers are the same for both maps.

Write

$$
g_{\lambda}(z)=\lambda^{2}+\sum_{n=1}^{\infty} c_{n} z^{n} .
$$

Our next goal is to estimate how close the fixed points of $f_{\lambda}$ and $g_{\lambda}$ are to each other. Those fixed points satisfy the equations

$$
\lambda= \pm i z \cdot \sqrt{f^{[2]}(z)}=: Q_{f}^{ \pm}(z)
$$

and

$$
\lambda= \pm i z \cdot \sqrt{g^{[2]}(z)}=: Q_{g}^{ \pm}(z)
$$

respectively. The subindex $\lambda$ is omitted from $f^{[2]}$ and $g^{[2]}$ since the tails do not depend on the constant term. 
By Lemma $5.1\left\|f-g_{0}\right\|_{r}<\delta$. Thus, by Lemma 2.1,

$$
\left\|f^{[2]}-g^{[2]}\right\|_{r}<\frac{4 \delta}{r^{2}}
$$

From (5.2) and Condition (C9) we get

$$
\left|g^{[2]}(z)\right|>\frac{4 \delta}{r^{2}}
$$

for all $z \in D_{r}$. Now, inequalities (5.2) and (5.3), together with Condition (C 3), allow us to apply Lemma 2.2 to $g^{[2]}$, and we obtain a branch $\sqrt{g^{[2]}}$ of the square root of $g^{[2]}$ such that

$$
\left\|\sqrt{f^{[2]}}-\sqrt{g^{[2]}}\right\|_{r}<\frac{2 \sqrt{\delta}}{r} .
$$

Therefore, using Condition (C10), we get

$$
\left\|Q_{f}^{ \pm}-Q_{g}^{ \pm}\right\|_{r}<2 \sqrt{\delta}<\frac{\sqrt{a_{2}} r}{4}=\frac{\left|a^{ \pm}\right| r}{4}
$$

where

$$
a^{ \pm}:= \pm i \sqrt{a_{2}} .
$$

With this notation, Condition (C4) states that

$$
\left\|\left(Q_{f}^{ \pm}\right)^{\prime}-a^{ \pm}\right\|_{r}<\frac{\left|a^{ \pm}\right|}{2} .
$$

In particular, Lemma 2.3 applies to $Q_{f}^{ \pm}$.

On the other hand, conditions (C5) - (C8) together imply

$$
\begin{aligned}
\left\|\left(Q_{g}^{ \pm}\right)^{\prime}-a^{ \pm}\right\|_{r} & =\sup _{z \in D_{r}}\left|\left[\sqrt{g^{[2]}(z)}+z \cdot\left(\sqrt{g^{[2]}(z)}\right)^{\prime}\right]-\sqrt{a_{2}}\right| \\
& <2 \frac{\sqrt{a_{2}}}{4}=\frac{\left|a^{ \pm}\right|}{2} .
\end{aligned}
$$

Thus, by (5.4), (5.5) and (5.6), we can apply Lemma 2.4 to obtain a bound on the inverses of $Q_{f}^{ \pm}$and $Q_{g}^{ \pm}$; i.e., on the functions $\chi_{f}^{ \pm}, \chi_{g}^{ \pm}$that represent the fixed points of $f_{\lambda}, g_{\lambda}$ in terms of $\lambda$. Namely, if we fix some $s>0$ such that $D_{s} \subset Q_{f}^{ \pm}\left(D_{r / 2}\right)$ (note that such $s$ exists and it does not depend on $g_{\lambda}$ ), then

$$
\left\|\chi_{f}^{ \pm}-\chi_{g}^{ \pm}\right\|_{s} \leq \frac{r}{2}
$$

In particular, the functions $\chi_{f}^{ \pm}$and $\chi_{g}^{ \pm}$belong to $\mathcal{A}_{s}$. As in the proof of Proposition 1.1 (compare (3.3)), we get

$$
\chi_{f}^{ \pm}(\lambda)= \pm \frac{i}{\sqrt{a_{2}}} \lambda+\frac{a_{3}}{2 a_{2}^{2}} \lambda^{2}+\lambda^{3} \cdot \chi_{f}^{ \pm[3]}(\lambda)
$$

and

$$
\chi_{g}^{ \pm}(\lambda)= \pm \frac{i}{\sqrt{c_{2}}} \lambda+\frac{c_{3}}{2 c_{2}^{2}} \lambda^{2}+\lambda^{3} \cdot \chi_{g}^{ \pm[3]}(\lambda) .
$$

Inserting the right-hand side of (5.9) as the argument in the formula for the derivative

yields the multipliers

$$
g_{\lambda}^{\prime}(z)=1+2 c_{2} z+3 c_{3} z^{2}+\left(z^{4} \cdot g_{\lambda}{ }^{[4]}(z)\right)^{\prime},
$$

$$
g_{\lambda}^{\prime}\left(\chi_{g}^{ \pm}(\lambda)\right)=1 \pm 2 i \sqrt{c_{2}} \lambda-\frac{2 c_{3}}{c_{2}^{2}} \lambda^{2}+\lambda^{3} \cdot \kappa(\lambda),
$$


where $\kappa$ is an analytic function with a rather complicated formula involving $c_{2}, c_{3}$, $c_{2}^{-1}$, the functions $g_{\lambda}^{[4]}, \chi_{g}^{ \pm[3]}$, and their first derivatives.

Now, Cauchy's estimate (2.1), together with Lemma 5.1] gives a uniform bound on $c_{2}$ and $c_{3}$, and Condition (C11) does a similar job with $c_{2}^{-1}$. Moreover, Lemma 5.1 and formula (5.7), together with the tail estimate (2.3), give uniform bounds for the functions $g_{\lambda}^{[4]}$ and $\chi_{g}^{ \pm[3]}$. Then, the global Cauchy estimate (2.2) gives uniform bounds for the derivatives $\left(g_{\lambda}^{[4]}\right)^{\prime}$ and $\left(\chi_{g}^{ \pm[3]}\right)^{\prime}$. All in all, these bounds imply that

$$
\|\kappa\|_{s}<C
$$

for some constant $C$ independent of $g$ and $\lambda$.

By the triangle inequality

$$
|| g_{\lambda}^{\prime}\left(\chi_{g}^{ \pm}(\lambda)\right)|-| 1 \pm 2 i \sqrt{c_{2}} \lambda-\frac{2 c_{3}}{c_{2}} \lambda^{2}||<C \lambda^{3}
$$

for all $\lambda \in[0, s]$ and $g \in \mathcal{P}_{r, \delta}(f)$. We have

$$
\begin{aligned}
\left|1 \pm 2 i \sqrt{c_{2}} \lambda-\frac{2 c_{3}}{c_{2}} \lambda^{2}\right|^{2} & \\
=\left(1+\frac{2 c_{3}}{c_{2}} \lambda^{2}\right)^{2}+\left(2 \sqrt{c_{2}} \lambda\right)^{2} & =1+4\left(c_{2}-\frac{c_{3}}{c_{2}}\right) \lambda^{2}+\frac{4 c_{3}^{2}}{c_{2}^{2}} \lambda^{4} \\
= & \left(1+2\left(c_{2}-\frac{c_{3}}{c_{2}}\right) \lambda^{2}\right)^{2}+4\left(2 c_{3}-c_{2}^{2}\right) \lambda^{4} .
\end{aligned}
$$

As before, all coefficients in this formula are bounded (in absolute value) by constants independent of $g \in \mathcal{P}_{\delta, r}(f)$, so

$$
\left|1 \pm 2 i \sqrt{c_{2}} \lambda-\frac{2 c_{3}}{c_{2}} \lambda^{2}\right|+\left(1+2\left(c_{2}-\frac{c_{3}}{c_{2}}\right) \lambda^{2}\right)
$$

is bounded away from 0 by such a constant. Similarly, $4\left(2 c_{3}-c_{2}^{2}\right)$ is also bounded by such a constant, so

$$
|| 1 \pm 2 i \sqrt{c_{2}} \lambda-\frac{2 c_{3}}{c_{2}} \lambda^{2}\left|-\left(1+2\left(c_{2}-\frac{c_{3}}{c_{2}}\right) \lambda^{2}\right)\right|<C_{1} \lambda^{4}
$$

for some constant $C_{1}$ independent of $g \in \mathcal{P}_{\delta, r}(f)$. Together with (5.10), this gives

$$
|| g_{\lambda}^{\prime}\left(\chi_{g}^{ \pm}(\lambda)\right)\left|-\left(1+2\left(c_{2}-\frac{c_{3}}{c_{2}}\right) \lambda^{2}\right)\right|<C_{2} \lambda^{3}
$$

for some constant $C_{2}$ independent of $g \in \mathcal{P}_{\delta, r}(f)$. Again, $c_{2}-c_{3} / c_{2}$ is bounded (in absolute value) by a constant independent of $g \in \mathcal{P}_{\delta, r}(f)$, so there is $\rho>0$ such that if $\lambda \in(0, \rho]$ and $g \in \mathcal{P}_{\delta, r}(f)$, then the sign of $\left|g_{\lambda}^{\prime}\left(\chi_{g}^{ \pm}(\lambda)\right)\right|-1$ is the same as the sign of $c_{2}-c_{3} / c_{2}$. Moreover, the sign of $c_{2}-c_{3} / c_{2}$ is the same as the sign of $a_{2}-a_{3} / a_{2}$ for all $g \in \mathcal{P}_{\delta, r}(f)$.

To apply this result to $g_{\Lambda}$, we need $\Lambda \leq \rho$. However, in view of Lemma 4.1 and the definition of $\Lambda$, this can be achieved by taking $\varepsilon$ sufficiently small (and independent of $\widetilde{f}$ ).

Since $g_{\Lambda}$ is conjugate to $\widetilde{f}$, the multipliers of their fixed points coincide, and the result follows. 


\section{REFERENCES}

[1] Núria Fagella and Antonio Garijo, The parameter planes of $\lambda z^{m} \exp (z)$ for $m \geq 2$, Comm. Math. Phys. 273 (2007), no. 3, 755-783. MR2318864

[2] John Milnor, Remarks on iterated cubic maps, Experiment. Math. 1 (1992), no. 1, 5-24. MR.1181083 (94c:58096)

[3] Michał Misiurewicz and Ana Rodrigues, Double standard maps, Comm. Math. Phys. 273 (2007), no. 1, 37-65. MR2308749 (2008b:37067)

[4] Shizuo Nakane and Dierk Schleicher, On multicorns and unicorns. I. Antiholomorphic dynamics, hyperbolic components and real cubic polynomials, Internat. J. Bifur. Chaos Appl. Sci. Engrg. 13 (2003), no. 10, 2825-2844. MR2020986 (2004i:37099)

[5] David Singer, Stable orbits and bifurcation of maps of the interval, SIAM J. Appl. Math. 35 (1978), no. 2, 260-267. MR0494306 (58:13206)

Department of Mathematical Sciences, iUpui, 402 N. Blackford Street, IndianapoLIS, INDIANA 46202-3216

E-mail address: mmisiure@math.iupui.edu

Department of Mathematical Sciences, iUpui, 402 N. Blackford Street, IndianapoLIS, INDIANA 46202-3216

E-mail address: rperez@math.iupui.edu 